\title{
Multispectral Quantum Dots-in-a-Well Infrared Detectors Using Plasmon Assisted Cavities
}

\author{
Rajeev V. Shenoi, Jessie Rosenberg, Thomas E. Vandervelde, Member, IEEE, Oskar J. Painter, and Sanjay Krishna
}

\begin{abstract}
We present the design, fabrication, and characterization, of multi-spectral quantum dots-in-a-well (DWELL) infrared detectors, by the integration of a surface plasmon assisted resonant cavity with the infrared detector. A square lattice and rectangular lattice cavity, formed by modifying the square lattice have been used in this design. By confining the resonant mode of the cavity to detector active region, the detector responsivity and detectivity have been improved by a factor of 5 . A spectral tuning of 5.5 to 7.2 $\mu \mathrm{m}$ has been observed in the peak response of the detectors, by tuning the lattice constant of the cavity. Simulations indicate the presence of two modes of absorption, which have been experimentally verified. The use of a rectangular lattice predicts highly polarization sensitive modes in $x$ - and $y$-direction, which are observed in fabricated detectors. A peak detectivity of $3.1 \times 10^{9}$ $\mathrm{cm} \cdot \sqrt{\mathrm{Hz}} / \mathrm{W}$ was measured at $77 \mathrm{~K}$. This design offers a cost-effective and simple method of encoding spectral and polarization information, in infrared focal plane arrays.
\end{abstract}

Index Terms-Focal plane array, long wavelength infrared (LWIR), multi-spectral detection, photodetector, quantum dots.

\section{INTRODUCTION}

$\mathbf{M}$ ULTI-SPECTRAL infrared (IR) detection has major applications in the field of gas detection, identification of chemical and biological compounds, remote sensing and thermography [1]. This capability is considered an integral part of third generation IR systems, along with large format arrays and higher temperature of operation [1]. Multi-spectral detection can help in detection of targets, especially in low contrast and high clutter. Current multi-spectral systems make use of an external filter with a broadband focal plane array (FPA) or disperse the light on to several arrays [2]. This approach, however increases the cost of the arrays several fold. The use of external components such as filters or beam splitters increase the size of these systems and require complex optical alignment, as array sizes increase. As a result current 30-50 band multi-spectral arrays cost $\$ 10-\$ 15$ million. A limited multi-spectral capability;

Manuscript received October 05, 2009; revised December 09, 2009. Current version published March 31, 2010. This work was supported by the AFOSR through its Nano Initiative Program and MURI Program in plasmonics, AFRL, and by the IC Post-Doctoral Program.

R. V. Shenoi and S. Krishna are with the Department of Electrical and Computer Engineering, Center for High Technology Materials, University of New Mexico, Albuquerque, NM 87106 USA (e-mail: rshenoi@ece.unm.edu; skrishna@chtm.unm.edu).

T. E. Vandervelde is with the Electrical and Computer Engineering Department, Tufts University, Medford, MA 02155 USA (e-mail: tvanderv@ece.tufts. edu).

J. Rosenberg and O. J. Painter are with the Department of Applied Physics, California Institute of Technology, Pasadena, CA 91125 USA (e-mail: jessier@caltech.edu; opainter@caltech.edu).

Digital Object Identifier 10.1109/JQE.2010.2042682 viz. dual color detection, can be achieved in arrays using back to back biased diodes [3], [4] or by asymmetric design of absorbing regions [5], enabling detection of multiple wavelengths by changing the bias polarity. Encoding of spectral and polarization information within the pixel can lead to large scale cost savings, reduced complexity and increased functionality of the arrays.

The mid-wave infrared (MWIR) and long-wave infrared (LWIR) regions are of great interest in imaging applications, due to the emission characteristics of objects near room temperature. Targets close to room temperature have high spectral content in these regimes and can be effortlessly identified using their spectral and polarization signatures. The major detector technologies currently existing in this regimes are bulk InSb in the MWIR, bulk HgCdTe in LWIR, and quantum well infrared photodetectors (QWIP) in LWIR. HgCdTe detectors have a high quantum efficiency and low dark currents; but due to problems inherent in the control of composition of the alloy, these detectors have a low yield. This increases the cost of $\mathrm{HgCdTe}$ detectors manyfold. InSb detectors are currently the dominant technology in MWIR, but need cooling to $77 \mathrm{~K}$ for their operation. Quantum dot infrared detectors (QDIP) and strained layer superlattices (SLS) are considered to be emerging technologies for third generation infrared detectors. These devices along with the QWIP, use transitions within quantum confined structures for infrared detection. They use mature growth and processing technologies associated with III-V compounds and this makes fabrication of large arrays using these technologies easier.

We have used a variant of the QDIP, known as the dots-in-a-well (DWELL) infrared photodetector as the technology to demonstrate spectral tuning [5]. In this technology quantum dots are embedded in a quantum well, and intersubband transitions in the conduction band of this system result in infrared absorption. The DWELL structure provides better control of the absorption wavelength when compared to QDIPs and provides a lower dark current. Dual color operation is also possible due to the nature of bound to bound and bound to continuum transitions that result in infrared absorption. As a result, the DWELL system exhibits a spectral response from 3-9 $\mu \mathrm{m}$. This is very relevant for demonstration of spectral tuning, as it provides a broad wavelength range over which response of the cavity can be tuned. The DWELL system, using asymmetrically placed dots in a well can result in bias tunable spectral response due to quantum confined Stark effect (QCSE). These designs have been successfully demonstrated in focal plane arrays of sizes up to $1024 \times 1024$ [6], [7].

Encoding of spectral information into a pixel has been attempted before by the use of Fabry-Perot cavities, which are 
epitaxially grown or by using microelectronic mechanical actuators (MEMS) [8], [9]. Another approach is to use gratings etched on to the surface of the detector. This has resulted in improving performance of QWIPs in the LWIR region. Gratings etched into the active region couple incident light into higher order modes enabling higher absorption [10], [11]. The use of dielectric and metallic deep etched photonic crystals have been attempted in the LWIR region [12], [13], but the high aspect ratio needed in etching makes processing difficult. Here we present a novel method whereby the spectral and polarization response of a DWELL detector is tuned by coupling the absorption of the active region to the resonant modes of a surface plasmon cavity integrated with the detector. This technique simplifies the processing required for the integration of the cavity without increasing the dark currents, is detector agnostic and can easily be transferred to a focal plane array.

\section{Growth AND Device Structure}

The detector samples used for demonstration of multi-spectral sensing, have InAs quantum dots embedded in quantum wells as their active region with GaAs substrates. This design offers considerable advantages over systems based on quantum dots alone. Typically, solid state quantum dots are grown in the Stranski-Krastanow mode, using self assembly techniques [14]. This method results in dots with varying sizes and hence it is difficult to control the operating wavelength of the detector. In a DWELL structure, transitions that result in infrared detection are from the ground state of the dot to a state in the well or in the continuum [5]. This results in better control of operating wavelength for the system. The DWELL structures also exhibit a dual color response, with one peak in the MWIR and the other in the LWIR regions. This is due to the transitions from a state in the dot to quantum well and from the dot to the continuum. Bias tunable spectral response can be obtained in a detector based on DWELL design. This is done by placing quantum dots asymmetrically in wells, resulting in QCSE, leading to asymmetric spectral response with applied bias polarity [15].

Two variants of the DWELL design were used to fabricate the detectors. The first structure consisted of quantum dots embedded in a single quantum well. The InAs dots were embedded in $\mathrm{In}_{0.15} \mathrm{Ga}_{0.85}$ As wells, with $\mathrm{GaAs}$ as the barrier material [15]. The strain introduced due to the growth of the well and the quantum dots, limits the number of stacks of active region to 15 layers. Hence an alternate design, known as the dots-in-a-double-well (DDWELL) was developed that uses InAs dots embedded in $\operatorname{In}_{0.15} \mathrm{Ga}_{0.85} \mathrm{As} / \mathrm{GaAs}$ wells, with $\mathrm{Al}_{0.10} \mathrm{Ga}_{0.90} \mathrm{As}$ as the barrier [16]. The primary well for this system is GaAs as opposed to $\mathrm{In}_{0.15} \mathrm{Ga}_{0.85}$ As in the previous design. The $\operatorname{In}_{0.15} \mathrm{Ga}_{0.85}$ As layers are very thin, with $1 \mathrm{~nm}$ above and below the dots. Reduction in the amount of strain inducing $\mathrm{In}_{0.15} \mathrm{Ga}_{0.85}$ As leads to growth of a higher number of active region stacks. The DDWELL design has 30 stacks of active region when compared to the DWELL design. This results in higher absorption cross-section of these devices, enabling their higher temperature operation. The use of DDWELL structures increases the operating temperature of the detectors, while maintaining its advantages like low dark current and spectral tuning. As to be discussed in Section III, the higher number

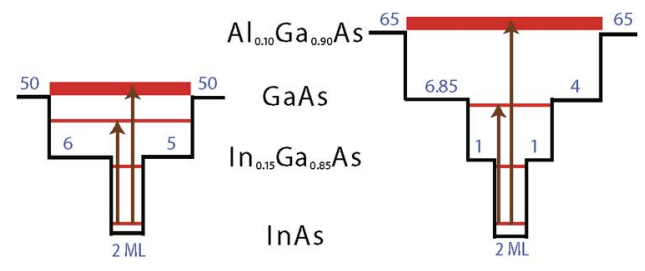

(a)

(b)

Fig. 1. Conduction band diagram of the DWELL and DDWELL designs. The dimensions of each layer are indicated in nm (blue) unless mentioned otherwise. The possible energy levels of the system (in red) and transitions resulting in infrared detection in the MWIR and LWIR windows are shown (in brown). The active region is $0.965 \mu \mathrm{m}$ thick in DWELL sample and $2.45 \mu \mathrm{m}$ in DDWELL sample. (a) DWELL. (b) DDWELL.

of active region stacks results in a higher modal confinement of the the resonant mode. A schematic representation of the conduction band of the DWELL design is shown in Fig. 1(a) and for the DDWELL design in Fig. 1(b).

The detector structures were grown using solid source molecular beam epitaxy (MBE) using a V80 machine. The active region is sandwiched between top and bottom contacts consisting of $\mathrm{n}$-doped GaAs, Si doped to $10^{18} / \mathrm{cm}^{-3}$. An $\mathrm{Al}_{0.70} \mathrm{Ga}_{0.30} \mathrm{As}$ layer exists below the bottom contact layer, serving as a cladding layer to confine the resonant mode energy in the active region.

\section{MODELING}

The cavity used here consists of a square lattice with square holes in it [17]. A schematic of the structure used for simulation is illustrated in Fig. 2(A). A waveguide formed by a thin metallic layer covering the aperture of the detector and bottom cladding layer helps to confine the energy of the mode withing the active region. The square pattern etched on the detector provides confinement in the lateral dimension. Finite difference time domain (FDTD) analysis was performed on this structure, assuming a $1 \%$ normal incidence single pass absorption for 15 stack DWELL and $2 \%$ for 30 stack DDWELL designs. These measurements agree well with the previously reported values of absorption measured on 30 stack DWELLs with similar structures [18]. The thickness of active regions were 0.965 and 2.45 $\mu \mathrm{m}$ for DWELL and DDWELL samples, respectively. The confinement of the fundamental mode within the active region was $42 \%$ for DWELL and $91 \%$ for the DDWELL samples. This is due to the higher active volume present in the DDWELL samples as a result of the thicker active region. A plot of index of refraction and mode profile of the fundamental mode of the system is shown on Fig. 2(B).

The TM bandstructure of the system is shown in Fig. 3(a) [19]. There is no bandgap in this structure. Instead, we concentrate on two orthogonal flatband modes at the $\Gamma$ point, to which free space waves easily couple. Low group velocity of these modes confines it in the active region, enabling higher absorption. The symmetry of the square lattice allows presence of two orthogonal modes, one polarized in the $x$-direction and the other in the $y$-direction. In a perfect square lattice, these modes are degenerate. They can be split, however, by breaking the symmetry of the lattice. The bandstructure for a system, where the lattice is stretched by $10 \%$ in one direction and compressed by $10 \%$ in the orthogonal direction is shown in Fig. 3(c). The two 

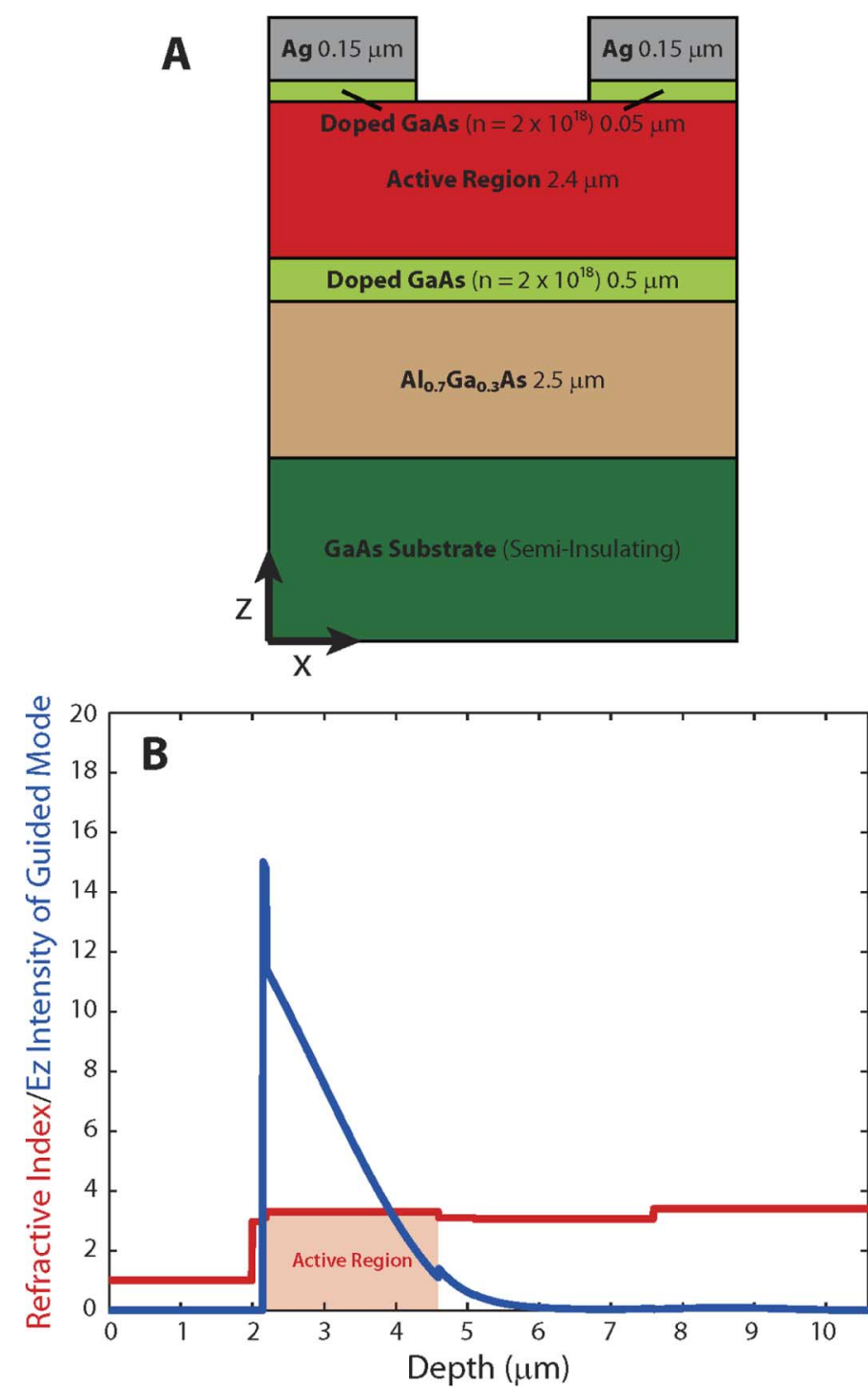

Fig. 2. Simulation of the plasmonic cavity: (A) Schematic of the simulated region. A $2 \%$ single pass absorption is assumed for the DDWELL samples and a $1 \%$ absorption is assumed for DWELL samples. (B) Mode profile of the fundamental mode for the DDWELL samples. A 91\% confinement is observed in the active region for this mode.

orthogonal modes split in frequency as a result of lattice asymmetry. These modes would be strongly polarization dependent, and detection of these modes can provide a scheme for encoding polarization into the pixel.

The plot of simulated active region absorption with wavelength is indicated in Fig. 4, for a lattice constant of $a=2.94 \mu \mathrm{m}$. The fundamental mode of the system can be observed at a wavelength of $9.6 \mu \mathrm{m}$ and a higher order mode is observed at $6.5 \mu \mathrm{m}$. A peak absorption of $11.5 \%$ was observed for the fundamental mode, indicating an enhancement factor of 5.7 with respect to a device without the cavity.

\section{Processing And Characterization}

Detector structures were processed in a class-100 cleanroom using standard techniques of mesa etching, passivation and contact metal deposition. The pixels consisted of top-illuminated $410 \mu \mathrm{m} \times 410 \mu \mathrm{m}$ square mesas, with aperture diameters ranging between 25 and $300 \mu \mathrm{m}$. A thin layer of $\mathrm{SiN}_{x}$ was deposited as a passivation layer, followed by a contact metal and a thin plasmonic metal (Ti/Ag) evaporation by e-beam deposition. The plasmon metal covers the entire aperture of the detector, eliminating the detector response to IR light at this point. Care was taken to cover all excess area on the device by metal to reduce substrate scattering. Following this, three sets of samples were fabricated using e-beam lithography and dry etching: Sample A with DWELL active region, sample B and sample $\mathrm{C}$ with the DDWELL active region. Sample A and $\mathrm{B}$ have periodic square holes in a square lattice pattern, while sample $\mathrm{C}$ had lattice constants stretched in one direction and compressed in the orthogonal direction. Two control samples without the cavity or the plasmon metal were also prepared, control1 for sample A and control2 for samples B and C, as they have the same active region, to compare the effect of the cavity on the detector. The patterned samples and the control sample were part of the same growth and were processed together till the plasmon metal step, to eliminate variations in growth and processing. A top view of the fabricated detector is shown in Fig. 5(A) and an SEM image of the fabricated lattice is shown in Fig. 5(B).

To characterize the detectors, the spectral response, signal, noise and dark current of the devices were measured. The spectral response was measured at various bias voltages using a Nicolet 6700 Fourier transform infrared spectrometer (FTIR). Spectral response of sample A for a bias of $-4.2 \mathrm{~V}$ is shown in Fig. 6 and of sample B at a bias of $5 \mathrm{~V}$ in Fig. 7, along with control samples at the same bias. The spectral response of patterned devices show the presence of resonant phenomena, showing a peak absorption for the detector at the peak of the resonant cavity. The spectra of sample A show a marked difference from the spectrum of the control sample. The spectra show two modes with peak wavelengths of the fundamental mode varying from 8.5 to $9 \mu \mathrm{m}$ and 6.1 to $6.6 \mu \mathrm{m}$ for the higher order mode, while the control sample shows a response with a peak at 10.3 $\mu \mathrm{m}$. The full width half maxima (FWHM) of the spectra is 900 $\mathrm{nm}$, indicating a $\mathrm{Q}$ factor of 10 . The additional peak observed in Fig. 6(c) may be due to the peel-off of the plasmon metal layer, coupling light to the active region through the exposed region. For sample B, by varying the lattice constant from 1.82 to $2.45 \mu \mathrm{m}$, the peak of the detector response has been tuned from 5.5 to $7.2 \mu \mathrm{m}$. The LWIR peak of $9.1 \mu \mathrm{m}$ present in the control sample is suppressed due to the effect of the cavity and the lower wavelength peak corresponding to cavity resonance has been enhanced. A higher order mode is also visible at lower wavelengths, as predicted by the simulations.

Spectral response from devices in sample $\mathrm{C}$, which has a rectangular lattice with square holes due to stretching and compression of the square lattice, is shown in Fig. 8(A) and (B). The device A) has a lattice constant of 2.1 in the $x$-direction and 2.6 $\mu \mathrm{m}$ in the $y$-direction while device B) has lattice constants of 1.8 and $2.1 \mu \mathrm{m}$ in $x$ - and $y$-directions, respectively. The detector response for unpolarized light and light polarized in orthogonal directions was measured using a wiregrid polarizer. The splitting of degenerate modes and their stronger response to light polarized in one direction can be observed. The mode in Fig. 8(A) at $8 \mu \mathrm{m}$ is $90^{\circ}$ polarized while the mode at $6.6 \mu \mathrm{m}$ is strongly 

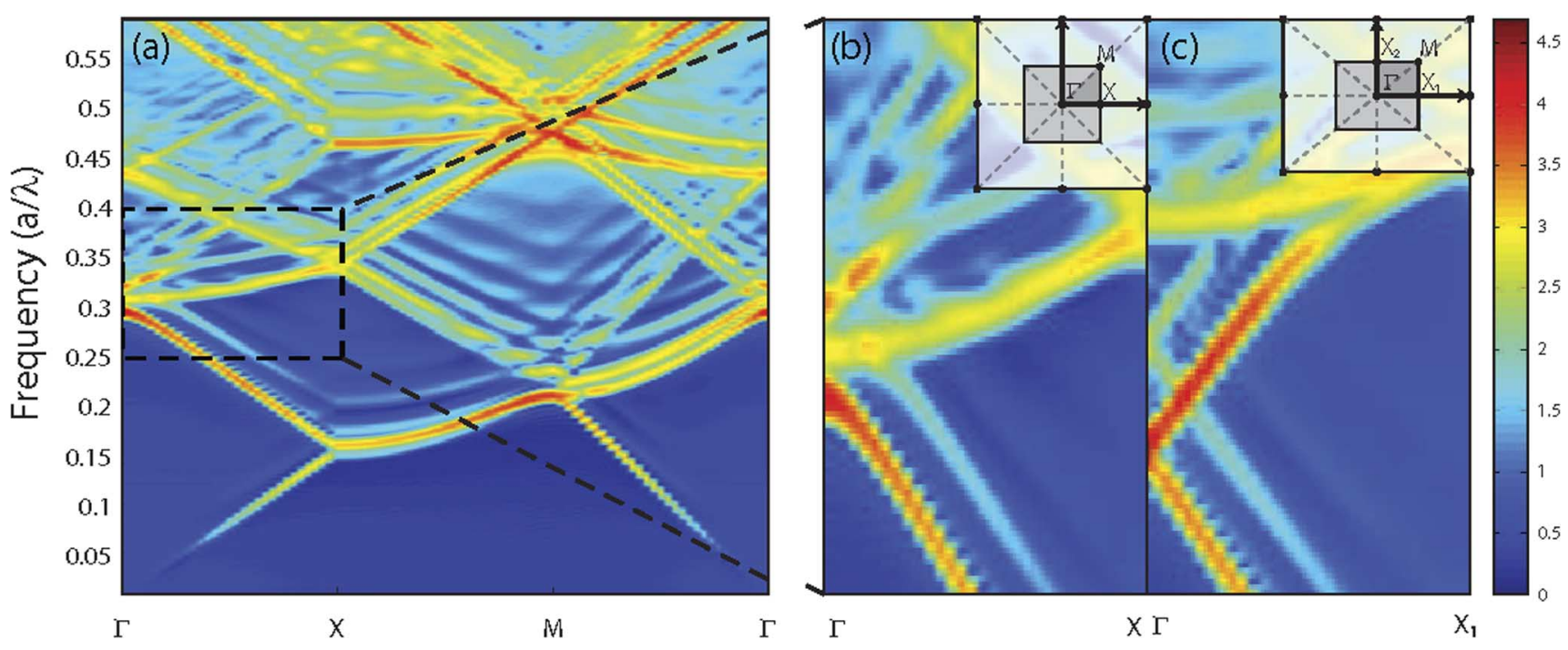

Fig. 3. (a) Bandstructure of the fabricated lattices: (b) Band profile near the $\Gamma$ point for the square lattice. The degenerate flatband modes can be observed. (c) Bandstructure of the stretched and compressed lattice at the $\Gamma$ point. The modes of (b) are split in frequency.

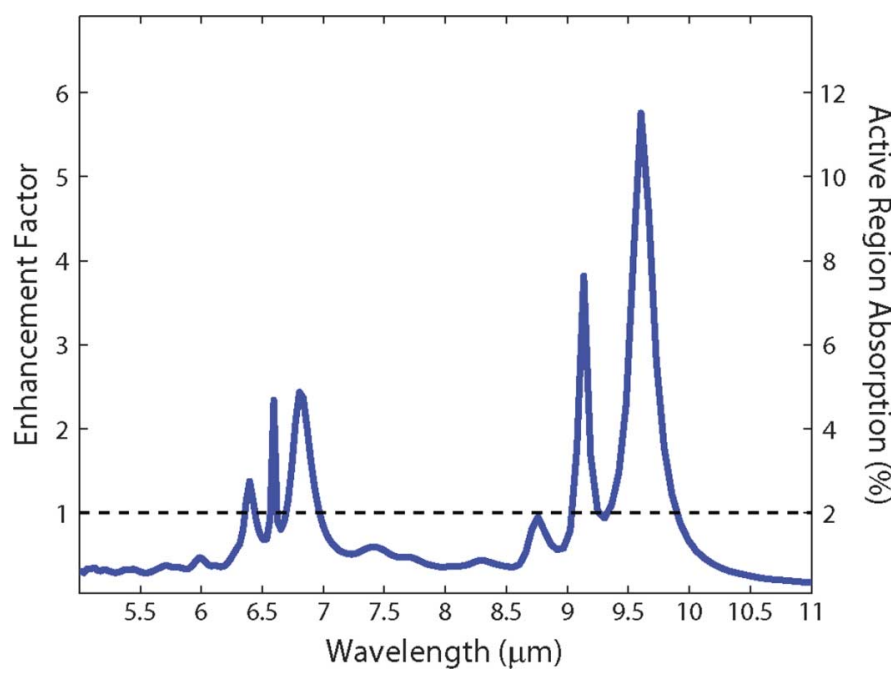

Fig. 4. Simulated absorption of the DDWELL detector with wavelength. A peak enhancement factor of 5.7 is predicted for the fundamental mode. A baseline is shown, indicating the single pass absorption of the DWELL region.

polarized with light at $0^{\circ}$ and has an intensity 2.26 times that of $90^{\circ}$ polarized light. In Fig. $8(\mathrm{~B})$ the mode at $6.6 \mu \mathrm{m}$ is $90^{\circ}$ polarized while the one at $5.5 \mu \mathrm{m}$ is $0^{\circ}$ polarized.

The signal and noise of devices at $77 \mathrm{~K}$ was measured by irradiating with a blackbody at $800 \mathrm{~K}$. From these measurements, the peak responsivity and detectivity can be extracted as described in [16]. These are plotted as a function of the applied bias in Fig. 9(A), for a representative device. The peak detectivity observed for this sample is $3.1 \times 10^{9} \mathrm{~cm} \cdot \sqrt{\mathrm{Hz}} / \mathrm{W}$ at $4 \mathrm{~V}$ bias, for a wavelength of $6.7 \mu \mathrm{m}$. The peak wavelengths of the patterned and control samples are different and hence for a fair comparison, their responsivity needs to be compared at the same wavelength. The enhancement factor is defined as

$$
E=\frac{R\left(\lambda_{i}\right)}{R_{\text {control }}\left(\lambda_{i}\right)}
$$

where $R\left(\lambda_{i}\right)$ is the responsivity of the patterned detector at the resonant wavelength and $R_{\text {control }}\left(\lambda_{i}\right)$ is the responsivity of the control sample at the same wavelength. The plasmonic detectors show an enhancement in detectivity and responsivity, when compared to the control sample. The observed enhancement factors for various wavelengths from samples $\mathrm{B}$ and sample $\mathrm{C}$ are shown in Fig. 9(B). The observed enhancement factors vary between 1.5 to 5.5 , with peak observed at $7.2 \mu \mathrm{m}$. This plot represents a conservative estimate for the enhancement factor, as substrate scattering is not totally eliminated in the control sample. The enhancement in responsivity and detectivity of the samples is not identical, due to the variations in noise between the patterned sample and the control sample. The difference in factors across various wavelengths may be attributed to the variations in DWELL absorption and processing of cavity. This enhancement is seen at wavelengths where the DWELL absorption peak is not prominent. The device performance can be further improved by aligning the resonant peak of the cavity with peak absorption of the device.

The dark currents of control sample and patterned devices were measured, to examine the effect of patterning the cavity. The average dark current density of the control samples and dark current densities from patterned devices is plotted in Fig. 10. The dark current of the control sample falls within the statistical distribution of dark currents in the patterned sample, which indicates that the patterning process has minimal contribution to the total dark current of the detector. The variations in dark current density may be due to the pixel to pixel variations in processing of detector and may not be due to the process of patterning the cavity. This method of integrating cavities minimizes the damage to the device, as the patterning is confined to the surface of the detector and does not etch the active region.

\section{CONCLUSION}

In conclusion, we have designed and fabricated a multispectral polarization sensitive DWELL and DDWELL detectors in MWIR and LWIR region. A surface plasmon resonant cavity 

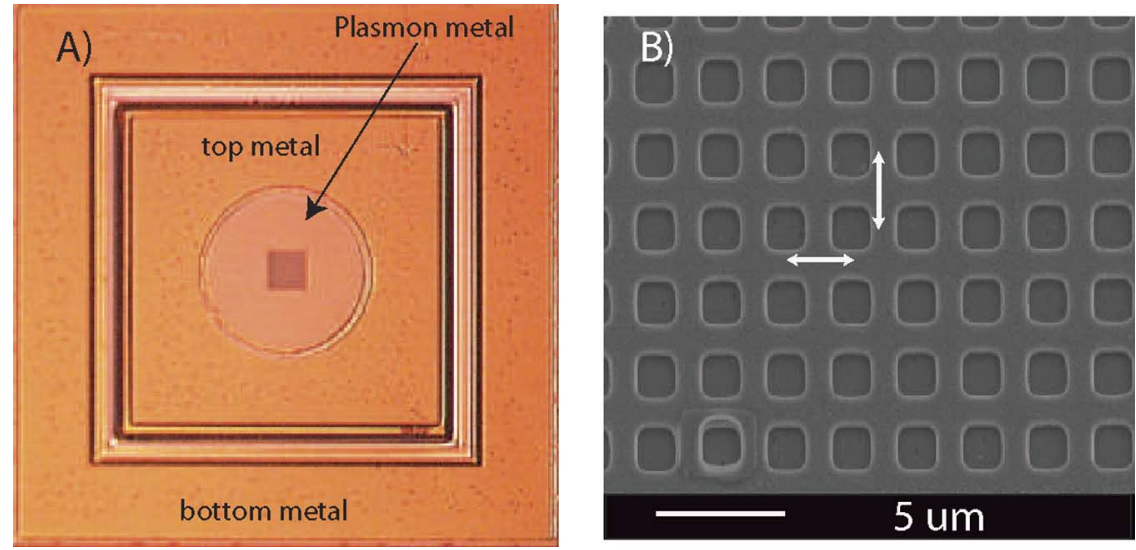

Fig. 5. Images of the fabricated plasmonic DWELL/DDWELL detector. (A) Optical image of the fabricated detector. The mesa, top metal, bottom metal and plasmonic metal can be observed. The square pattern at the center is the fabricated pattern. (B) Scanning electron microscopy (SEM) image of the etched pattern in the detector aperture. The pattern is a rectangular lattice with square holes, formed from a square lattice by stretching lattice constants in one direction and compressing it in the orthogonal direction.

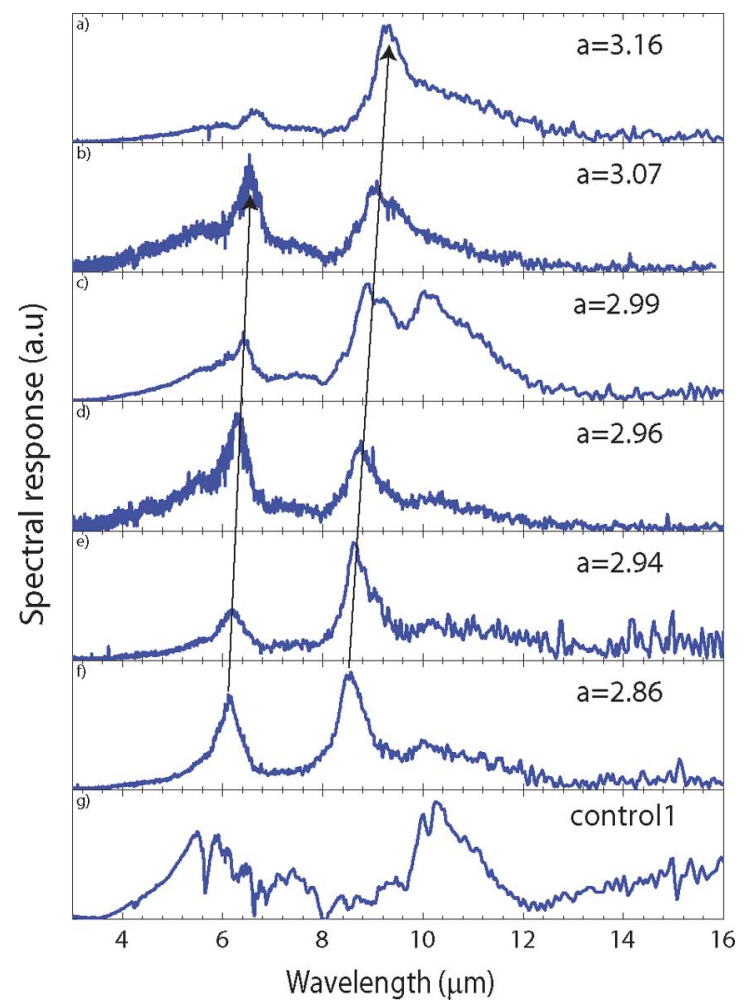

Fig. 6. Spectral response from sample A (DWELL material, square lattice) obtained at $30 \mathrm{~K},-4.2 \mathrm{~V}$ bias. Two modes of the resonant cavity, whose peak wavelengths increase with the lattice constant can be seen.

was designed and integrated with a DWELL/DDWELL infrared detector. The absorption of the detector is coupled to resonant modes of the cavity. The peak of the detector response has been tuned, from 5.5 to $7.2 \mu \mathrm{m}$ and from 8.5 to $9 \mu \mathrm{m}$, by changing the lattice constant of the fabricated pattern. Modeling of the device predicted two resonant modes for the cavity, which were observed in detector response and are in good agreement with the predictions. The symmetry of the square lattice, when broken by stretching and compressing the lattice in two orthogonal directions, resulted in splitting of the otherwise degenerate modes in frequency. These resonant modes were observed to be strongly

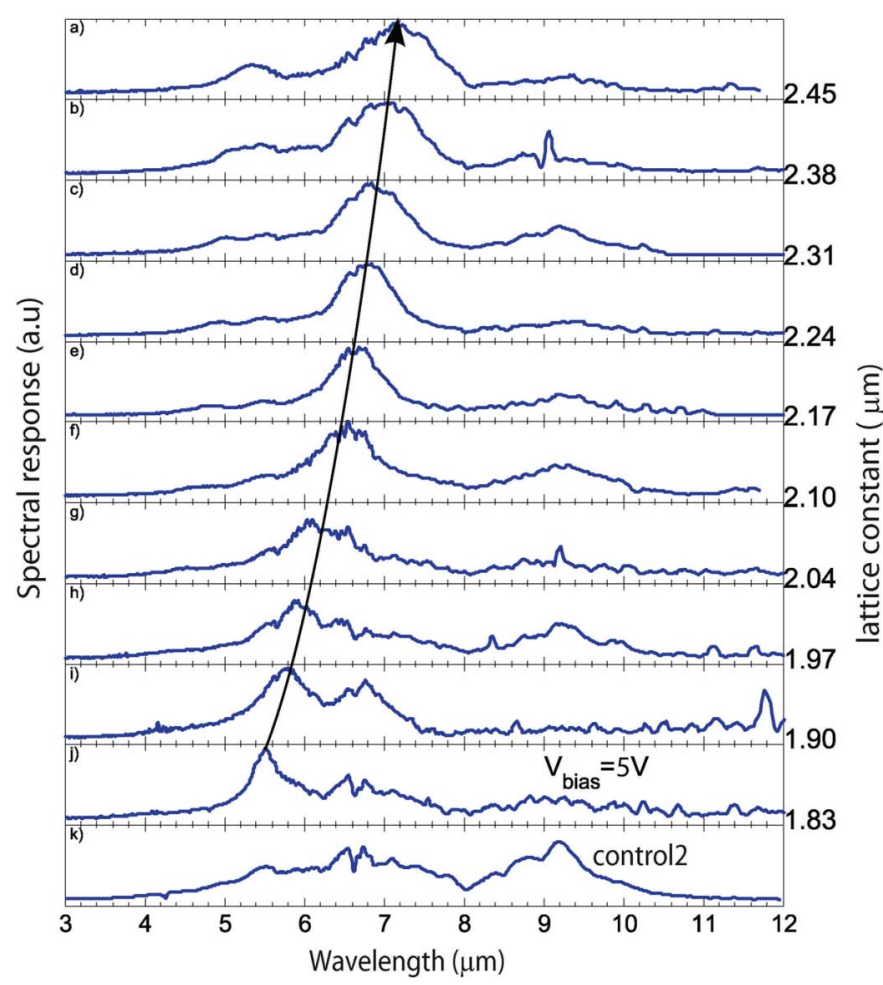

Fig. 7. Normalized spectral response from sample B, measured at $30 \mathrm{~K}$ indicating tuning of peak wavelength with the lattice constant. The devices were biased at a voltage of $5 \mathrm{~V}$, except for plot i) which was biased at $3 \mathrm{~V}$. Peak wavelength variation from 5.5 to $7.2 \mu \mathrm{m}$ is achieved by changing the lattice constant from 1.82 to $2.45 \mu \mathrm{m}$. A two point smoothing filter has been applied on the spectra.

polarization dependent. Due to the coupling of the cavity resonance to the detector, an improvement in detectivity and responsivity of the detectors could be observed. A peak detectivity of $3.1 \times 10^{9} \mathrm{~cm} \cdot \sqrt{\mathrm{Hz}} / \mathrm{W}$ was measured at $4 \mathrm{~V}$ bias. Dark current measurements indicate that the patterning does not result in a significant increase in dark current. The processing technique used to integrate the cavity on to the detector is simple, as it does not involve removal of material from the detector, is detector agnostic and can be easily transferred to a FPA. This design can 

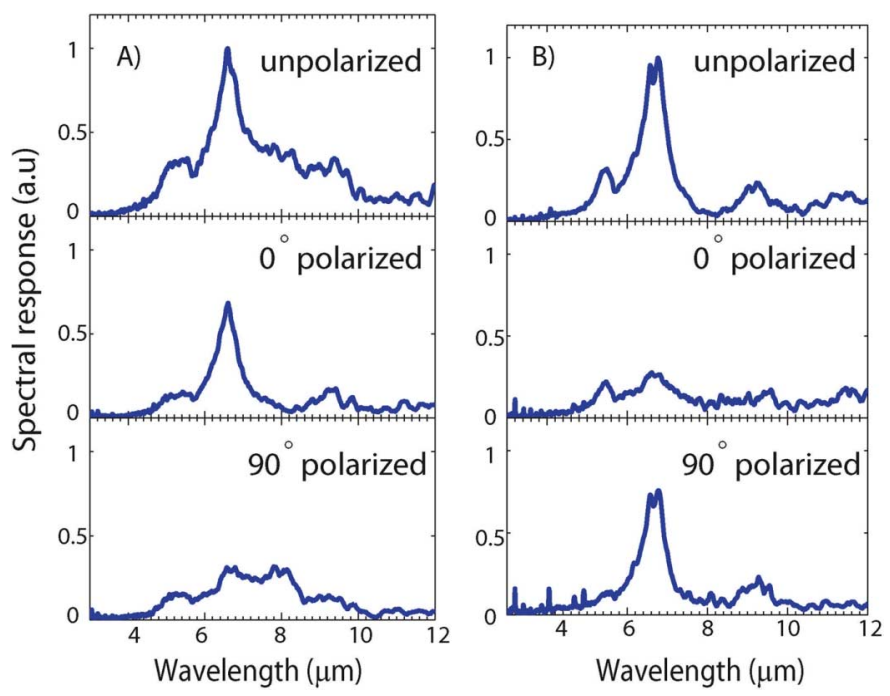

Fig. 8. Polarization dependent spectral response from devices in sample $\mathrm{C}$ (DDWELL material, stretched lattice), at $5 \mathrm{~V}, 30 \mathrm{~K}$. Response to unpolarized light, light polarized at $0^{\circ}$ and $90^{\circ}$. Polarization sensitive modes at different wavelengths, due to different lattice constants in orthogonal directions can be observed. The resonant mode is dominated by $0^{\circ}$ polarized light in (A) and by $90^{\circ}$ polarized light in $(\mathrm{B})$.
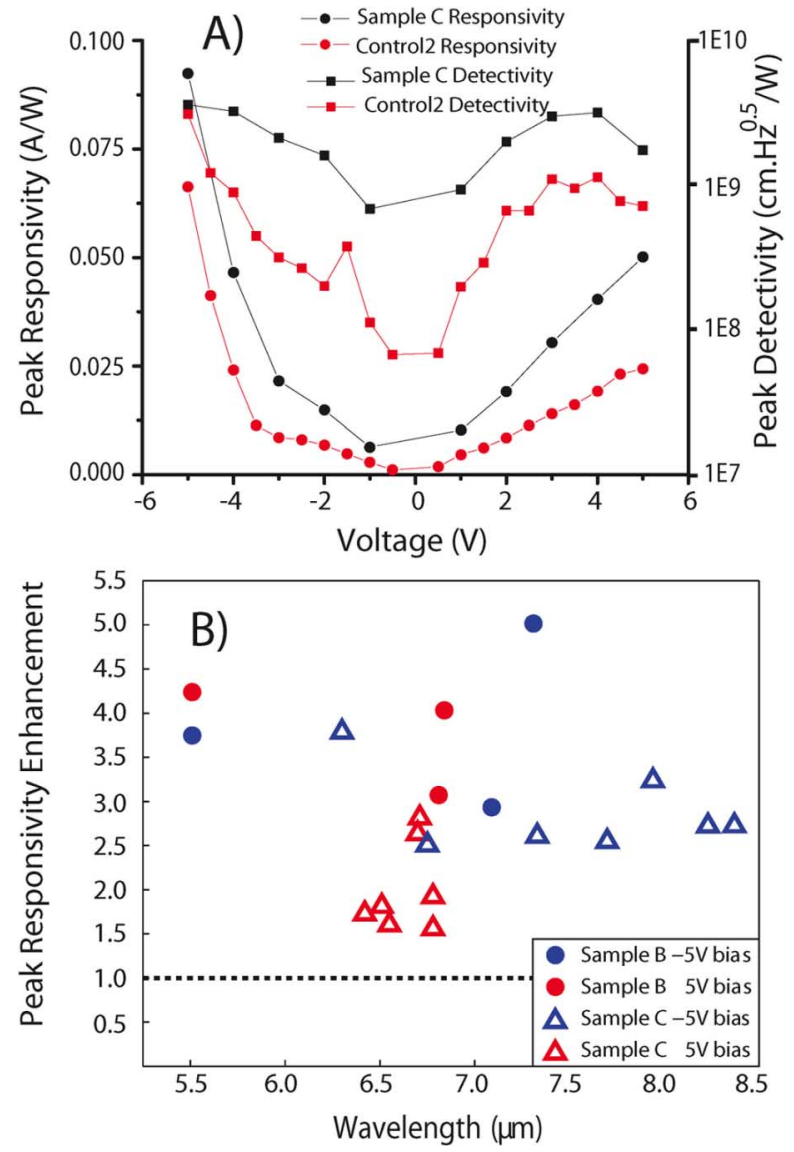

Fig. 9. Measured responsivity and detectivity of processed detectors. (A) Responsivity and detectivity of a detector from sample $\mathrm{C}$ at $77 \mathrm{~K}$, with that of the control sample. The peak wavelength of the control sample at positive and negative bias voltages is $9.4 \mu \mathrm{m}$ and $8.7 \mu \mathrm{m}$ respectively. The device from sample $\mathrm{C}$ has a peak wavelength of $6.7 \mu \mathrm{m}$. (B) Measured enhancement factors of detectors across various samples, for a bias voltage of -5 and $5 \mathrm{~V}$ at $77 \mathrm{~K}$. A peak enhancement is observed at $7.2 \mu \mathrm{m}$.

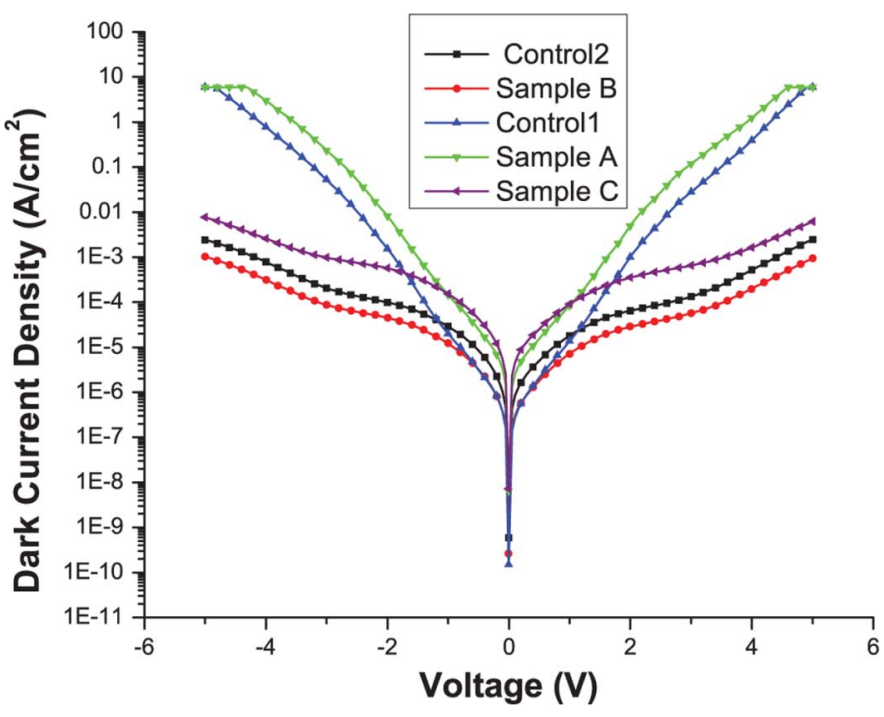

Fig. 10. Dark current measurements at $77 \mathrm{~K}$ for patterned and control samples. The average dark current density for control 1 and control 2 and representative detectors from samples A, B, and C are shown.

result in encoding of spectral and polarization information in the FPA pixel, with one additional lithography step, and can eliminate complex optics currently in use for multispectral FPAs.

\section{ACKNOWLEDGMENT}

The authors would like to thank T. Nelson for helpful discussions, R. Perahia for help in the detector fabrication, J. S. Brown for the growth, and S. J. Lee for discussions during characterization.

\section{REFERENCES}

[1] A. Rogalski, J. Antoszewski, and L. Faraone, "Third-generation infrared photodetector arrays," J. Appl. Phys. vol. 105, no. 9, p. 091101, 2009. [Online]. Available: http://link.aip.org/link/?JAP/105/091101/1

[2] M. A. Kinch, Fundamentals of Infrared Detector Materials. Bellingham, WA: SPIE Press, 2007.

[3] M. Z. Tidrow, J. C. Chiang, S. S. Li, and K. Bacher, "A high strain two-stack two-color quantum well infrared photodetector," Appl. Phys. Lett. vol. 70, no. 7, pp. 859-861, 1997. [Online]. Available: http://link. aip.org/link/?APL/70/859/1

[4] R. Rehm, M. Walther, J. Schmitz, J. Fleißner, J. Ziegler, W. Cabanski, and R. Breiter, "Dual-colour thermal imaging with InAs/GaSb superlattices in mid-wavelength infrared spectral range," Electron. Lett., vol. 42, pp. 2124-2127, 2006.

[5] S. Krishna, "Quantum dots-in-a-well infrared photodetector," J. Phys. $D$, vol. 38, pp. 2142-, 2005.

[6] E. Varley, M. Lenz, S. J. Lee, J. S. Brown, D. A. Ramirez, A. Stintz, and S. Krishna, "Single bump, two-color quantum dot camera," Appl. Phys. Lett., vol. 91, pp. 081120-, 2007.

[7] D. Z.-Y. Ting, S. V. Bandara, S. D. Gunapala, J. M. Mumolo, S. A. Keo, C. J. Hill, J. K. Liu, E. R. Blazejewski, S. B. Rafol, and Y.-C. Chang, "Submonolayer quantum dot infrared photodetector," Appl. Phys. Lett. vol. 94, no. 11, pp. 111107-, 2009. [Online]. Available: http://link.aip. org/link/?APL/94/111107/1

[8] R. S. Attaluri, J. Shao, K. T. Posani, S. J. Lee, J. S. Brown, A. Stintz, and S. Krishna, "Resonant cavity enhanced InAs/ $/ \mathrm{In}_{0.15} \mathrm{Ga}_{0.85} \mathrm{As}$ dots-in-a-well quantum dot infrared photodetector," J. Vac. Sci. Tech. B., vol. 25, pp. 1186-, 2007.

[9] C. Musca, J. Antoszewski, K. Winchester, A. Keating, T. Nguyen, K. Silva, J. Dell, L. Faraone, P. Mitra, J. Beck, M. Skokan, and J. Robinson, "Monolithic integration of an infrared photon detector with a mems-based tunable filter," IEEE Electron Device Lett., vol. 26, no. 12 , pp. 888-890, Dec. 2005 
[10] T. R. Schimert, S. L. Barnes, A. J. Brouns, F. C. Case, P. Mitra, and L. T. Claiborne, "Enhanced quantum well infrared photodetector with novel multiple quantum well grating structure," Appl. Phys. Lett. vol. 68, no. 20, pp. 2846-2848, 1996. [Online]. Available: http://link.aip. org/link/?APL/68/2846/1

[11] S. Schartner, S. Golka, C. Pflugl, W. Schrenk, A. M. Andrews, T. Roch, and G. Strasser, "Band structure mapping of photonic crystal intersubband detectors," Appl. Phys. Lett., vol. 89, pp. 151107-, 2006.

[12] K. T. Posani, V. Tripathi, S. Annamalai, N. R. Weisse-Bernstein, S. Krishna, R. Perahia, O. Crisafulli, and O. J. Painter, "Nanoscale quantum dot infrared sensors with photonic crystal cavity," Appl. Phys. Lett., vol. 88, p. 151104, 2006.

[13] R. V. Shenoi, D. A. Ramirez, Y. Sharma, R. S. Attaluri, J. Rosenberg, O. J. Painter, and S. Krishna, "Plasmon assisted photonic crystal quantum dot sensors," presented at the Soc. Photo-Opt. Instrumentation Eng. (SPIE) Conf. Series, Oct. 2007. [Online]. Available: http://dx.doi. org/10.1117/12.735724, ser. Presented at the Society of Photo-Optical Instrumentation Engineers (SPIE) Conference, vol. 6713

[14] D. Bimberg, M. Grundmann, and N. N. Ledentsov, Quantum Dot Heterostructures. New York: Wiley, 1999.

[15] S. Krishna, S. Raghavan, G. von Winckel, A. Stintz, G. Ariyawansa, S. G. Matsik, and A. G. U. Perera, "Three-color $\left(\lambda_{p 1} \approx 3.8 \mu \mathrm{m}, \lambda_{p 2} \approx\right.$ $8.5 \mu \mathrm{m}$, and $\lambda_{p 3} \approx 23.2 \mu \mathrm{m}$ ) InAs/InGaAs quantum-dots-in-a-well detector," Appl. Phys. Lett., vol. 83, pp. 2746-, 2003.

[16] R. V. Shenoi, R. S. Attaluri, A. Siroya, J. Shao, Y. D. Sharma, A. Stintz, T. E. Vandervelde, and S. Krishna, "Low-strain InAs/InGaAs/GaAs quantum dots-in-a-well infrared photodetector," J. Vac. Sci. Tech. B., vol. 26, pp. 1136-1139, 2008

[17] J. Rosenberg, R. V. Shenoi, T. E. Vandervelde, S. Krishna, and O. Painter, "A multi-spectral and polarization-selective surface-plasmon resonant mid-infrared detector," Appl. Phys. Lett., submitted for publication.

[18] S. D. Gunapala, S. V. Bandara, C. J. Hill, D. Z. Ting, J. K. Liu, S. B. Rafol, E. R. Blazejewski, J. M. Mumolo, S. A. Keo, S. Krishna, Y. C. Chang, and C. A. Shott, " $640 \times 512$ pixels long-wavelength infrared quantum-dot infrared photodetector imaging focal plane array," IEEE J. Quantum Electron., vol. 43, no. 3, pp. 230-237, Mar. 2007.

[19] J. Rosenberg, R. V. Shenoi, T. E. Vandervelde, S. Krishna, and O. Painter, "Design of plasmonic photonic crystal resonant cavities for polarization sensitive infrared photodetectors," Opt. Exp., submitted for publication.

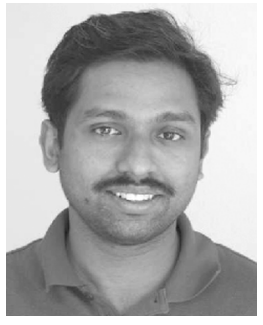

Rajeev V. Shenoi received the B.Tech. degree in electronics and communication engineering from the National Institute of Technology, Calicut, India, in 2002, and the Master's degree in electrical engineering from Indian Institute of Technology Madras, Chennai, India, in 2005 . He is currently pursuing the Ph.D. degree from the University of New Mexico, Albuquerque.

His research interests include infrared detectors and systems, plasmons, and fiber optic communication systems and devices.

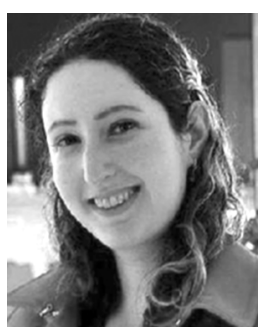

Jessie Rosenberg was born in Washington, DC, in 1986. She received the B.A. degree in physics (with Honors) from Bryn Mawr College, Bryn Mawr, PA, in 2004. She is currently pursuing the Ph.D. degree in applied physics from the California Institute of Technology, Pasadena.

Her research interests include plasmonics and photonic crystals, as well as harnessing the interactions between optical and mechanical excitations for physical and technological applications.

Ms. Rosenberg was a recipient of the National Science Foundation Graduate Research Fellowship in 2005.

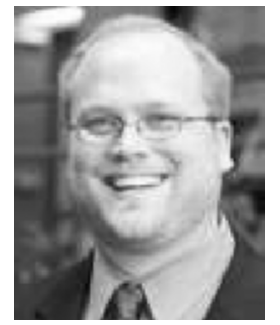

Thomas E. Vandervelde (M'01) earned two B.S. degrees from the University of Massachusetts, Amherst, one in physics and the other in astronomy, both in 1999, and the M.A. and Ph.D. degrees in physics from the University of Virginia, Charlottesville, in 2001 and 2004, respectively.

He presently holds the John A. and Dorothy M Adams Endowed-Chair Professorship as an Assistant Professor with the Electrical and Computer Engineering Department, Tufts University, Medford, MA. Prior to his present employment, he was a Research Assistant Professor of electrical and computer engineering with the Center for High Technology Materials, University of New Mexico, in 2007-2008 and he was a Post-Doctoral Fellow with University of New Mexico with S. Krishna in 2007, University of Illinois Urbana-Champaign with M. Feng in 2005-2006, and University of Virginia with M. Skrutskie in 2004-2005. Additionally, he also was a Visiting Assistant Professor of physics with Washington and Lee University in 2004-2005. He has authored/coauthored over 20 peer-reviewed journal articles, over 30 conference presentations, and has one provisional patent. His current and previous research interests are group IV and III-V materials for photodetectors, photovoltaics/thermophotovoltiacs, nanophysics/devices, terahertz electronics, and hetero-integrated circuits. Dr. Vandervelde was on the executive committee for the Albuquerque IEEE LEOS chapter from 2007-2008.

Dr. Vandervelde is a member of APS, SPS, AAS, Sigma Xi, MRS, TMS, AVS, AAAS, and Eta Kappa Nu.

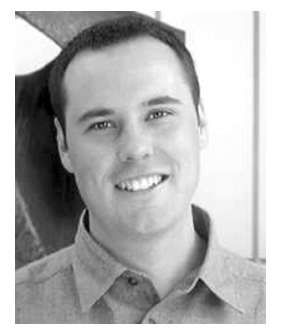

Oskar Painter received the B.S.E.E. degree from the University of British Columbia, BC, Canada, in 1994 the Master's degree in science and the Ph.D. degree in electrical engineering from the California Institute of Technology, Pasadena, in 1995 and 2001, respectively

In 2000, he helped found Xponent Photonics, an optical start-up company developing surface-mount photonics for telecom and data networking applications. In 2002, he returned to the California Institute of Technology as an Assistant Professor in applied physics. His general research interests lie in studying new and interesting ways in which light behaves within micro- and nano-scale dielectric and metallic structures. Currently, specific areas of research include: semiconductor cavity QED, integrated microphotonic and microwire "atom-chips", surface-plasmon physics and devices, and cavity optomechanics.

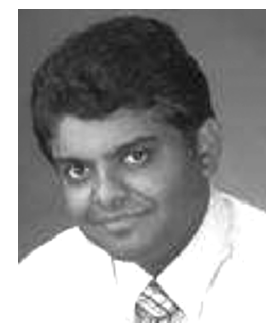

Sanjay Krishna received the Master's degree in physics from the Indian Institute of Technology (IIT), Madras, India, in 1996, the M.S. degree in electrical engineering and the $\mathrm{Ph} . \mathrm{D}$. degree in applied physics from the University of Michigan, Ann Arbor, in 1999 and 2001, respectively.

$\mathrm{He}$ is an Associate Professor of electrical and computer engineering with the Center for High Technology Materials, University of New Mexico. He joined the University of New Mexico as a tenure track faculty member in 2001 . His present research interests include growth, fabrication and characterization of self-assembled quantum dots and type II InAs/InGaSb based strain layer superlattices for mid infrared detectors. He has authored/coauthored over 70 peer-reviewed journal articles, over 40 conference presentations, and two book chapters, and has two issued and five pending patents.

Dr. Krishna was a recipient of the Gold Medal from IIT, Madras, India, in 1996, the Best Student Paper Award at the 16th NAMBE Conference in Banff, Canada, in 1999, the 2002 Ralph E. Powe Junior Faculty Award from Oak Ridge Associated Universities, the 2003 IEEE Outstanding Engineering Award, 2004 Outstanding Researcher Award from the ECE Department, the 2005 School of Engineering Junior Faculty Teaching Excellence Award, the 2007 North American Molecular Beam Epitaxy Young Investigator Award, the 2007 NCMR-DIA Chief Scientist Award for Excellence, and the 2008 Early Career Achievement Award from the SPIE and the IEEE Nanotechnology Council. 